\title{
Radioisotopes and Their Biomedical Applications
}

Nida Tabassum Khan*

Department of Biotechnology, Balochistan University of Information Technology Engineering and Management Sciences, Quetta, Pakistan

\begin{abstract}
Radionuclides also termed as radioisotopes are elements that possess radioactivity. It means upon decay they emit radiations like alpha, beta or gamma particles and transformed their nuclei to a stable state. This decaying property of radioisotopes is called half-life. Thus radioisotopes could be used for numerous biomedical purposes such as cancer and tumour treatment, imaging, biochemical assays, biological labelling, sterilization, clinical diagnostics, radioactive dating etc.
\end{abstract}

Keywords: Urinalysis; Bioassay; Tracer; Carbon dating

\section{Introduction}

Radioisotopes are widely used for a number of purposes following are some major applications of radioisotope.

\section{Biochemical analysis}

Biochemical assays are used to detect the presence and absence of radioisotopes. Therefore radioactive isotopes are used to label biological molecules. Such assays estimate the concentration of different constituents of plasma, body fluids, urine, blood etc. This technique is called radioimmuno-assays. An example is iodine bioassay which uses gamma emitters' radionuclides of Iodine-125 and Iodine-131 that accrues inside thyroid. Therefore gamma detector can be used to quantify the iodine content (uptake and intake) of the person's thyroid. The amount of measured radioiodine in the thyroid is compared with the Annual Limit on Intake (ALI) [1].

\section{Urinalysis}

Radioisotopes are eliminated from the body in body fluids. By determining the active contents in urine one can analyse the uptake and intake of a specific radionuclide [1].

\section{Tracer studies}

Radioisotope is used for biological labelling of cells or entities for identification or tracing specific molecules in an organism. S 35 P 32 and I 125 are widely used radioisotopes used for labelling [2].

\section{Carbon dating}

Radioactive carbon-14 decay could be used to estimate the age of organic materials. For example carbon dating revealed that the burial cloth of Jesus Christ originated during the medieval times between A.D. 1260-1390. Similarly mummified remains found frozen in the Italian Alps were at least 5000 years old [3].

\section{Potassium dating}

Radio potassium-40 decays to stable $40 \mathrm{Ar}$. Thus, by measuring relative ratio of $40 \mathrm{~K}$ and $40 \mathrm{Ar}$ in rocks enable us to determine the age of rocks since its formation [4].

\section{Clinical diagnostic}

Positron Emission Tomography (PET) and PETCT make use of radionuclides emitting positron particle that is injected in to the target cell or tissue. Radionuclide decay release positron particles which interact with the nearby negatively charged particle resulting in the emission of gamma rays which is detected by a PET or gamma camera to give an exact image of the target [5].

\section{Radionuclide Therapy (RNT)}

This therapy makes use of radioisotopes that emits radiations upon their decay. These emitted radiations are used to target specific cancerous cells, tumours etc. to control their abnormal growth or completely eradicate it. For example cobalt- 60 is use as a source of gamma radiation for radionuclide therapy, gamma knife radiosurgery and brachytherapy. Similarly targeted alpha therapy uses alphaemitting radionuclide such as Bi-213, Lead-212, and Boron-10 to for treating pancreatic, ovarian and melanoma cancers [6].

\section{Sterilizing}

Sterilization of surgical instruments such as syringes, gloves, clothing and instruments using gamma mitting radionuclides including Cobalt-60, Cs-137 etc. [7].

\section{Radiopharmaceuticals}

Incorporation of radioisotopes to biologically active substances is introduced into body in order to observe the functioning of an organ functioning or a metabolic path way etc. For example Yttrium-90 and Iodine-131 is used as radiopharmaceuticals for the treatment of non-Hodgkin's lymphoma and hyperthyroidism respectively (Table 1) $[8]$.

\section{Conclusion}

Radioisotopes are used for numerous medical purposes which marks it potential in the field of medical science.

*Corresponding author: Nida Tabassum Khan, Department of Biotechnology, Balochistan University of Information Technology Engineering and Management Sciences, Quetta, Pakistan, Tel: 03368164903; E-mail: nidatabassumkhan@yahoo.com

Received July 27, 2017; Accepted August 03, 2017; Published August 12, 2017

Citation: Khan NT (2017) Radioisotopes and Their Biomedical Applications. J Biomol Res Ther 6: 156. doi:10.4172/2167-7956.1000156

Copyright: (c) 2017 Khan NT. This is an open-access article distributed under the terms of the Creative Commons Attribution License, which permits unrestricted use, distribution, and reproduction in any medium, provided the original author and source are credited. 


\begin{tabular}{|c|c|c|}
\hline Isotope & Half-life & Medical uses \\
\hline Ac-225 & $10.0 \mathrm{~d}$ & Cancer treatment \\
\hline Ac-227 & $21.8 y$ & Cancer treatment \\
\hline Am-241 & $432 y$ & Detection of osteoporosis \\
\hline As-72 & 26.0h & PET/ SPECT \\
\hline As-74 & $17.8 \mathrm{~d}$ & In biomedical \\
\hline At-211 & $7.21 \mathrm{~h}$ & Cancer treatment \\
\hline Au-198 & $2.69 d$ & Cancer treatment \\
\hline B-11 & Stable & Tumor treatment \\
\hline $\mathrm{Be}-7$ & $53.2 d$ & Used in berylliosis (chronic lung disease) studies \\
\hline $\mathrm{Bi}-212$ & 1.10h & Cancer treatment, cellular dosimetry studies \\
\hline $\mathrm{Bi}-213$ & $45.6 \mathrm{~m}$ & Cancer treatment \\
\hline $\mathrm{Br}-75$ & $98 \mathrm{~m}$ & SPECT/PET \\
\hline $\mathrm{Br}-77$ & $57 \mathrm{~h}$ & Monoclonal antibody labeling \\
\hline $\mathrm{C}-11$ & $20.3 \mathrm{~m}$ & Radiotracer in PET scans \\
\hline C-14 & $5730 y$ & Radiolabeling for tumors detection \\
\hline Cd-109 & $462 d$ & Cancer detection, pediatric imaging \\
\hline Ce-139 & $138 d$ & Calibration of germanium gamma detectors \\
\hline Ce-141 & $32.5 d$ & $\begin{array}{l}\text { diagnosis of gastrointestinal tract, myocardial blood } \\
\text { flow measurement }\end{array}$ \\
\hline Cf-252 & $2.64 y$ & Cancer treatment. \\
\hline Co-55 & $17.5 \mathrm{~h}$ & Planar and SPECT /PET imaging \\
\hline Co-57 & $272 d$ & $\begin{array}{l}\text { In radiotracer, source for X-ray fluorescence } \\
\text { spectroscopy }\end{array}$ \\
\hline Co-60 & $5.27 y$ & Teletherapy, disinfectant, radiotherapy \\
\hline Cr-51 & $27.7 \mathrm{~d}$ & Radiolabeling and dosimetry \\
\hline Cs-130 & $29.2 \mathrm{~m}$ & Myocardial localizing agent \\
\hline Cs-131 & $9.69 d$ & Intracavity implants for radiotherapy \\
\hline Cs-137 & $30.2 y$ & Blood irradiators, PET imaging, and tumor treatment \\
\hline \multirow[t]{2}{*}{ Cu-61 } & $3.35 \mathrm{~h}$ & Planar imaging, SPECT or PET \\
\hline & & Positron emitting radionuclide \\
\hline Cu-62 & $4.7 \mathrm{~m}$ & Tracer, PET/SPETC imaging \\
\hline Cu-64 & $12.7 \mathrm{~h}$ & Cancer treatment \\
\hline Cu-67 & $61.9 \mathrm{~h}$ & $\begin{array}{c}\text { Cancer treatment/diagnostics, radio immunotherapy, } \\
\text { SPECT or PET }\end{array}$ \\
\hline Dy-165 & $2.33 \mathrm{~h}$ & Radiation synovectomy, rheumatoid arthritis treatment \\
\hline Eu-152 & $13.4 y$ & Medical uses \\
\hline Eu-155 & $4.73 y$ & Osteoporosis detection \\
\hline $\mathrm{F}-18$ & $110 m$ & Radiotracer, imaging \\
\hline Fe-55 & $2.73 y$ & Heat source \\
\hline Fe-59 & $44.5 d$ & Medical use \\
\hline Ga-64 & $2.63 \mathrm{~m}$ & $\begin{array}{l}\text { Treatment of pulmonary diseases ending in fibrosis of } \\
\text { lungs. detection of Hodgkin's/non-Hodgkins lymphoma }\end{array}$ \\
\hline Ga-67 & $78.3 \mathrm{~h}$ & osteomyelitis detection \\
\hline Ga-68 & $68.1 \mathrm{~m}$ & Imaging, detection, treatment of pancreatic cancer \\
\hline Gd-153 & $242 d$ & Photon source, detection, imaging \\
\hline Ge-68 & $271 d$ & Imaging \\
\hline $\mathrm{H}-3$ & $12.3 y$ & Radiolabeling, imaging \\
\hline $\mathrm{I}-122$ & $3.6 \mathrm{~m}$ & Neurology studies \\
\hline $\mathrm{I}-123$ & $13.1 \mathrm{~h}$ & Imaging, cancer treatment \\
\hline \multirow[t]{2}{*}{$\mathrm{I}-124$} & $4.17 d$ & Tracer, imaging \\
\hline & & $\begin{array}{l}\text { Detection of osteoporosis, } \\
\text { imaging, tracer, brain } \\
\text { cancer treatment, } \\
\text { radiolabeling, interstitial }\end{array}$ \\
\hline $\mathrm{I}-125$ & $59.9 d$ & radiation therapy \\
\hline $\mathrm{I}-131$ & $8.04 d$ & $\begin{array}{c}\text { Tumor treatment, antibody labeling, radio } \\
\text { immunotherapy, cellular dosimetry, SPECT imaging, } \\
\text { treatment of prostate cancer }\end{array}$ \\
\hline $\mathrm{I}-132$ & $2.28 \mathrm{~h}$ & Mapping of areas \\
\hline $\ln -111$ & $2.81 d$ & $\begin{array}{c}\text { Detection of transplant rejection, imaging, labeling, } \\
\text { treatment of tumors }\end{array}$ \\
\hline In-115m & $4.49 \mathrm{~h}$ & Radiolabelling \\
\hline
\end{tabular}

\begin{tabular}{|c|c|c|}
\hline Ir-191m & $6 s$ & Cardiovascular angiography \\
\hline Ir-192 & $73.8 d$ & treatment of cancers \\
\hline $\mathrm{Kr}-81 \mathrm{~m}$ & $13.3 \mathrm{~s}$ & Lung imaging \\
\hline Lu-177 & $6.68 d$ & Heart disease treatment), cancer therapy \\
\hline $\mathrm{Mn}-51$ & $46.2 \mathrm{~m}$ & Myocardial localizing agent \\
\hline$M n-52$ & $5.59 d$ & PET scanning \\
\hline Mo-99 & $65.9 \mathrm{~h}$ & Organ imaging \\
\hline $\mathrm{N}-13$ & $9.97 \mathrm{~m}$ & PET imaging, myocardial perfusion \\
\hline $\mathrm{Nb}-95$ & $35 d$ & Myocardial tracer, PET imaging \\
\hline $0-15$ & $122 \mathrm{~s}$ & PET imaging / SPECT imaging \\
\hline Os-191 & $15.4 d$ & Cardiovascular angiography \\
\hline Os-194 & $6.00 y$ & Cancer treatment \\
\hline P-32 & $14.3 d$ & Cancer treatment, imaging, radiolabeling \\
\hline P-33 & $25 d$ & Labeling \\
\hline $\mathrm{Pb}-203$ & $2.16 \mathrm{~d}$ & Planar imaging, SPECT or PET, cellular dosimetry \\
\hline $\mathrm{Pb}-212$ & $10.6 \mathrm{~h}$ & Radioactive labelling, cellular dosimetry \\
\hline Pd-103 & $17 d$ & Prostate cancer treatment \\
\hline Pd-109 & $13.4 \mathrm{~h}$ & Potential radio therapeutic agent \\
\hline $\mathrm{Pu}-238$ & $2.3 y$ & Pacemaker \\
\hline Ra-223 & $11.4 \mathrm{~d}$ & Cancer treatment \\
\hline Ra-226 & $1.60 \mathrm{e} 3 \mathrm{y}$ & Target isotope to make Ac-227, Th-228, Th-229 \\
\hline $\mathrm{Rb}-82$ & $1.27 \mathrm{~m}$ & $\begin{array}{l}\text { Myocardial imaging agent, PET imaging, blood flow } \\
\text { tracers }\end{array}$ \\
\hline $\operatorname{Re}-186$ & $3.9 d$ & - \\
\hline $\operatorname{Re}-188$ & $17 \mathrm{~h}$ & Cancer treatment \\
\hline Rh-105 & $35.4 \mathrm{~h}$ & Potential therapeutic applications, radiolabeling \\
\hline Ru-97 & $2.89 d$ & Monoclonal antibodies labelling, imaging \\
\hline Ru-103 & $39 d$ & Radiolabeling, imaging \\
\hline S-35 & $87.2 d$ & Radiolabeling, cellular dosimetry \\
\hline Sc-46 & $84 d$ & Imaging \\
\hline \multirow{2}{*}{ Sc-47 } & \multirow{2}{*}{$3.34 d$} & Treatment of cancer/diagnostics \\
\hline & & Radio immunotherapy \\
\hline Se-72 & $8.4 d$ & Brain imaging, immunotherapy \\
\hline Se-75 & $120 \mathrm{~d}$ & Radiotracer \\
\hline Si-28 & Stable & Radiation therapy of cancer \\
\hline Sm-145 & $340 d$ & Brain cancer treatment \\
\hline Sm-153 & $2.00 d$ & Cancer treatment/diagnostics \\
\hline Sn-117m & $13.6 \mathrm{~d}$ & Pain relief \\
\hline Sr-85 & $65.0 \mathrm{~d}$ & Detection and imaging \\
\hline Sr-89 & $50 d$ & Cancer treatment, cellular dosimetry \\
\hline Sr-90 & $29.1 y$ & Immunotherapy \\
\hline Ta-178 & $9.3 \mathrm{~m}$ & Imaging \\
\hline Ta-179 & $1.8 \mathrm{y}$ & Source of X-ray \\
\hline Ta-182 & $115 d$ & Urinary cancer treatment \\
\hline Tb-149 & $4.13 \mathrm{~h}$ & Cancer treatment \\
\hline Tc-96 & $4.3 \mathrm{~d}$ & Animal studies \\
\hline Tc-99m & $6.01 \mathrm{~h}$ & nuclear imaging \\
\hline Th-228 & $720 d$ & Cancer treatment \\
\hline Th-229 & $7300 y$ & cancer treatment \\
\hline TI-201 & $73.1 \mathrm{~h}$ & Imaging, cellular dosimetry \\
\hline Tm-170 & $129 d$ & Treatment of cancers \\
\hline Tm-171 & $1.9 y$ & Medical uses \\
\hline W-188 & $69.4 d$ & Treatment of tumors \\
\hline \multirow{2}{*}{ Xe-127 } & \multirow{2}{*}{$36.4 d$} & Neuroimaging \\
\hline & & Lung studies \\
\hline $\mathrm{Xe}-133$ & $5.25 d$ & Lesion detection \\
\hline $\mathrm{Y}-88$ & $107 d$ & Cancer tumor therapy \\
\hline Y-90 & $64 \mathrm{~h}$ & $\begin{array}{c}\text { cellular dosimetry, treating rheumatoid arthritis and } \\
\text { cancers }\end{array}$ \\
\hline Y-91 & $58.5 d$ & Tumor treatment, dosimetry \\
\hline Yb-169 & $32 d$ & Clinical diagnostics \\
\hline
\end{tabular}




\begin{tabular}{|c|c|c|}
\hline Zn-62 & $9.22 \mathrm{~h}$ & Study of neurology \\
\hline $\mathrm{Zn}-65$ & $244 \mathrm{~d}$ & Medical uses \\
\hline $\mathrm{Zr}-95$ & $64.0 \mathrm{~d}$ & Medical uses \\
\hline
\end{tabular}

Table 1: Lists of radionuclides and their biomedical applications [9-48]

\section{References}

1. Friedlander G, Kennedy JW, Macias ES, Miller JM (1981) Nuclear and Radiochemistry. John Wiley \& Sons, NY, USA

2. Link JM, Krohn KA, Eary JF, Kishore R, LewellenTK, et al. (1986) 89Zr for antibody labeling and positron tomography. J Labelled Compd Radiopharm 23: 1296-1297.

3. Goh, KM (1991) Carbon dating. Carbon Isotope Techniques 1: 125

4. Dalrymple GB, Lanphere MA (1969) Potassium-argon dating: Principles, techniques and applications to geochronology. In (Freeman) San Francisco, California, p: 258.

5. Rosch F, Qaim SM, Stocklin G (1993) Production of the positron emitting radioisotope $86 \mathrm{Y}$ for nuclear medical application. Appl Radiat Isot 44: 677-681.

6. Wagner HN,Wiseman GA, Marcus CS (2002) guidelines for Radio immunotherapy for non-Hodgkin's lymphoma with 90Y-labeled anti-CD20 monoclonal antibody. J Nucl Med 43: 267-272.

7. Bakri A, Mehta K, Lance DR (2005) Sterilizing insects with ionizing radiation. In Sterile Insect Technique, Springer Netherlands, pp: 233-268.

8. Hosain Fazle (1996) Radiopharmaceuticals: therapeutic applications. 100 Years of X-rays and Radioactivity Bhabha Atomic Research Centre, Mumbai, India, pp: 447-461.

9. Zuckerman JD, Sledge CB, Shortkroff S, Venkatesan P (1987) Treatment of rheumatoid arthritis using radiopharmaceuticals. Nucl Med Biol 14: 211-218.

10. Davis MA, Chinol M (1989) Radiopharmaceuticals for radiation synovectomy, Evaluation of two yttrium-90 particulate agents. J Nucl Med 30: 1047-1055.

11. Pandey U, Mukherjee A, Chaudhary PR, Pillai MRA, Venkatesh M (2001) Preparation and studies with 90Y-labelled particles for use in radiation synvectomy. Appl Radiat Isot 55: 471-475.

12. Hsieh BT, Ting G, Hsieh HT, Shen LH (1993) Preparation of carrier free 90Y for medical applications by solvent extraction chromatography. Appl Radiat Isot 44: $1473-1480$

13. Mantravadi RVP, Spigos DG, Tan WS, Felix EL (1982) Intraarterial yttrium-90 in the treatment of hepatic malignancy. Radiology 142: 783-787.

14. Pandey U, Mukherjee A, Sarma HD, Das T, Pillai MRA, et al. (2002) Evaluation of 90Y-DTPA and 90Y-DOTA for potential application in intra-vascular radionuclide therapy. Appl Radiat Isot 57: 313-318.

15. Lenarczyk M, Goddu SM, Dandamudi VR, Howell RW (2001) Biologic dosimetry of bone marrow: Induction of micronuclei in reticulocytes after exposure to 32P and 90Y. J Nucl Med 42: 162-169.

16. Beyer GJ, Bergmann R, Kampf G, Mading P, Rosch F, et al. (1992): Simultaneous study of the biodistribution of radio-yttrium complexed with EDTMP and citrate ligands in tumour-bearing rats. Nucl Med Biol 19: 201-205.

17. Dejesus OT, Nickless RJ (1990) Production and purification of $89 \mathrm{Zr}$, a potential PET antibody label. Appl Radiat Isot 41: 789-790.

18. Goerner W, Noll B, Spies H, Thieme K (1988) Determination of technetium in 99Mo/99mTc-generators. J Radioanal Nucl Chem 122: 291-298.

19. Chervu LR, Lee HB, Goyal Q, Bloufox MD (1977) Use of 99mTc-Cu-DTPA complex as a renal function agents. J Nucl Med 18: 62-66.

20. Marchi A, Uccelli L, Bonardi M, Gallorini M, Groppi F, et al. (1995) Technetium complexes with ligands of pharmacological interest. J Radioanal Nucl Chem Art 195: 237-242.

21. Neirinckx RD, Canning LR, Piper IM, Nowotnik DP, Picket RD, et al. (1987) A new radiopharmaceutical for SPECT imaging of regional cerebral blood perfusion. J Nucl Med 28: 191-192.

22. Vallabhajosula S, Zimmerman RE, Picard M, Stritzke P, Mena I (1989) Technetium-99m-ECD a new brain imaging agent, In vivo kinetics and biodistribution studies in normal human subjects $\mathrm{J}$ Nucl Med 30: 599-604.

23. Kothari K, Banerjee S, Sharma HD, Pillai MRA (2000) Synthesis and radiolabeling of cysteine-oximes and pharmacological behaviour of their 99mTc complexes. Appl Radiat Isot 52: 69-76.

24. Pankaj P, Sen IB, Gupta A, Bembey S, Baruah B, et al. (2001) Localization of an infected cyst in adult polysistic kidney by Tc-99m HMPAO labeled leucocyte scan. Ind Nucl Med 16: 138-140.

25. Ding HJ, Shiau YC, Tsai SC, Wang JJ, Ho ST, (2002) Uptake of 99mTc tetrofosmin in lymphoma cell lines: a comparative study with $99 \mathrm{mTc}$ sestamibi. Appl Radiat Isot 56: 853-856.

26. Kao CH, Hsieh JF, Tsai SC, HoYJ, Changlai SP, et al. (2001) Paclitaxel-based chemotherapy for non small cell lung cancer:Predicting the response with 99mTc-tetrofosmin chest imaging. J Nucl Med 42: 17-20.

27. Holman BL (1976) Radionuclide Methods in evaluation of Myocardial Ischemia and infarction. Circulation 53: 112-118.

28. Umezawa H (1965) Bleomycin antitumour antibiotics of high molecular weight Antimicrob Agents Chemother 5: 1079-1085

29. Bonadonna G, Delena M, Monfardini S, Bartoli C, Bajetta E, et al. (1972) Clinical trial with bleomycin in lymphomas and in solid tumours. Eur $\mathrm{J}$ Cancer 8: 205-215.

30. Benjamin PP (1969) A rapid and efficient method of preparing 99mTc-serum albumin, Its clinical applications. Int J Appl Radiat Isot 20: 187-194.

31. Lin MS, Weber PM, Winchel HS (1972) Renal imaging in humans with the technetium labelled polypeptide cascidin. J Nucl Med 13: 517-521.

32. Lin MS, Goodwin DA, Kruse SL (1974) Bleomycin as a $99 \mathrm{mTc}$ carrier in tumor visualisation. J Nucl Med 15: 338-342.

33. Lin TH, Khentigan AA, Winchel AS (1974) 99mTc-chelate for organoradiomercuria renal agents. J Nucl Med 15: 34-35.

34. Hauser W, Atkins HL, Welson KG (1970) Technetium 99m DTPA: A new rsdiopharmaceutical for brain and kidney scanning. Radiology 94: 679-684.

35. Subramanian G, Mcafee JG, Mchter A (1973) 99mTc stannous phylate: A new in vivocolloid for imaging the reticuloendothelial system. J Nucl Med 14: 459-463.

36. Bekerman C, Gottschalk A (1971) Diagnostic significance of the relative uptake of liver compared to spleen in $99 \mathrm{mTC}$-sulfur colloid scintigraphy. J Nucl Med 12: $237-240$.

37. Baldas J, Bonnyman J (1985) Effect of the Tc-nitrido group on the behaviour of 99mTc-radiopharmaceuticals. Int J Appl Radiat Isot 36: 919-923.

38. Zhang J, Wang X (2001) Preparation of 99mTcN(CBDTC)2 and its biodistribution in mice. Appl Radiat Isot 55: 453-456.

39. Zhang C, Wang Y, Tian H, Yin D (2002) Preparation of 103Pd seed-molecular plating of 103Pd onto silver rod. Appl Radiat Isot 57: 309-311.

40. Wistow BW, Subramanium G, Van Heertum R (1977) An evaluation of 99m Tc-labelled hepatobilliary agents. J Nucl Med 18: 455-461.

41. Schaffer EA, Mcormond P, Dugan H (1980) Quantitative cholescintigraphy: assesment of gallblader filling and empty and duodenogastric reflux. Gastroenterology 79: 899-906.

42. Krishnamurthy GT, Bobba VR, Kingston E (1981) Radionuclide ejection fraction: a technique for quantitative analysis of motor function of the human gallbladder. Gastroenterology 80: 482-490.

43. Gambhir SS, Hawkins RA, Huang SC, Hall TR, Busuttil RW, et al. (1989) Trace kinetic modelling approaches for the quantification of hepatobillary function with technetium-99m DISIDA and scintigraphy. J Nuc Med 30: 1507-1518.

44. Mushtaq A, Pervez SH, Haider I, Mansur MS, Jehangir M (2000) A freez dried kit 99mTc(V)dimercaptosuccinic acid. J Radioanal Nucl Chem 243: 827-829.

45. Wang $X(2000)$ Synthesis and bio distribution of the $99 \mathrm{mTCN}-\mathrm{DMSA}$ complex as a potential bone imaging agent. J Radioanal Nucl Chem 243: 827-829.

46. Rosch F, Novgoredov AF, Qaim SM (1994) Thermochromatographic separation of $94 \mathrm{mTc}$ from enriched molybdenum targetsand its large scale production for nuclear medical application. Radiochim Acta 64: 113-120.

47. Rösch F, Qaim SM (1993) Nuclear data relevant to the production of the positron emitting technetium isotope $94 \mathrm{mTc}$ via the $94 \mathrm{Mo}(p, n)$ reaction. Radiochim Acta 62: 115-121.

48. Groshar D, Slobodin G, Zuckerman E (2002) Quantitatio of liver and spleen uptake of 99mTc-phytate colloid using SPECT: Detection of liver cirrhosis. J Nucl Med 43: 312-317. 